\title{
Evidencia y teoría del caso. Una relación sin resolver en la criminalística
}

\author{
Máximo Emilio Jordan Porras \\ mjordan2@utmachala.edu.ec
}

Fernando Alexis Ramírez González

framirez2@utmachala.edu.ec

Armando Rogelio Durán Ocampo

aduran@utmachala.edu.ec

Universidad Técnica de Machala

Ecuador

\section{RESUMEN}

El manejo de la evidencia física como aspecto importante dentro de los estudios de criminalística, ha sido muy debatido dentro de la literatura científica, sin embargo, pese a los intentos realizados en el proceso de construcción de este elemento fundamental dentro de los estudios de criminalística, existen todavía imprecisiones en el establecimiento de los protocolos necesarios para el correcto manejo de pruebas en el contexto específico de los hechos criminales; así como en el tratamiento posterior encaminado a que los objetos obtenidos como parte del estudio pericial, pasen a ser pruebas dentro de una audiencia de juicio oral, o sencillamente fortalecer la verosimilitud de una investigación y en consecuencia de la teoría del caso. Desde estos precedentes se realiza una investigación cuyo objetivo es contribuir a la divulgación de la importancia del manejo pertinente de las evidencias físicas en el trabajo pericial, defendiendo varias ideas una de las cuales considera que tras un hecho delictivo puede existir una multiplicidad de causas por lo que las evidencias físicas pueden ser variadas y su esclarecimiento debe partir de la confluencia entre distintas disciplinas científicas, precisándose de un buen planteamiento de evidencias para una adecuada teoría del caso.

Palabras clave: evidencia física; criminalística; manejo de pruebas; trabajo pericial; teoría del caso 


\title{
Evidence and theory of the case. An unsolved relationship in criminology
}

\begin{abstract}
The handling of physical evidence as an important aspect in criminology studies has been highly debated in the scientific literature, however, despite the attempts made in the process of building this fundamental element in criminology studies, there are still inaccuracies in the establishment of the necessary protocols for the correct handling of evidence in the specific context of the criminal acts; as well as in the subsequent treatment aimed at the objects obtained as part of the expert study, becoming evidence within an oral trial hearing, or simply strengthening the plausibility of an investigation and consequently of the theory of the case. From these precedents, an investigation is carried out whose objective is to contribute to the dissemination of the importance of the pertinent handling of physical evidence in the expert work, defending several ideas, one of which considers that after a criminal act there may be a multiplicity of causes for what that the physical evidence can be varied and its clarification must start from the confluence between different scientific disciplines, requiring a good approach to evidence for an adequate theory of the case.
\end{abstract}

Keywords: physical evidence; criminology; evidence handling; expert work; case theory

Artículo recibido: 15 noviembre. 2021 Aceptado para publicación: 10 diciembre 2021 Correspondencia: armandduran71@gmail.com

Conflictos de Interés: Ninguna que declarar 


\section{INTRODUCCIÓN}

La Criminalística es, sin lugar a dudas, una de las ciencias que más contribuye a que se realice una eficaz administración de la justicia. Esta disciplina pudo trascender al estatus de ciencia gracias a la aplicación del método científico en los procesos que realiza, y es precisamente la posibilidad de estudiar los resultados tangibles y observables que cauterizan a los eventos delictivos, la característica que más aporta a la explicación de la calidad de los hechos, otorgando a sus indicios el carácter de prueba.

No se puede negar el hecho de que dentro del marco del Derecho es la Criminalística, la ciencia que más contribuye a la obtención de las pruebas y evidencias, directamente desde el escenario en el cual se han producido los hechos. Ccaza (2012), expresa que la Criminalística aporta un conjunto de conocimientos que se convierten en una importante herramienta teórico metodológica de gran utilidad para diferentes esferas del quehacer humano, por su valor probatorio.

Es indudable que la Criminalística permite a los peritos forenses hacer entrega a la administración de justicia las evidencias probatorias, sin cuya participación le sería muy difícil a los magistrados demostrar los hechos. Este carácter auxiliar de la Criminalística, no la lleva a un plano secundario, por el contrario, apoya activamente al proceso jurídico aportando los medios que llevan a los operadores de justicia a reunir la evidencia necesaria (ya sea como información pertinente o evidencia física), necesarias para develar el trasfondo de los hechos e impartir justicia.

Pese a lo planteado existen autores como Elbert (1988) que desconocen el carácter de ciencia de la Criminalística, relegándola al carácter de disciplina auxiliar, aduciendo que no posee métodos propios y reduciéndola al papel de herramienta interpretativa. En su descargo puede plantearse que, reconoce que en la actualidad gran parte de la comunidad científica le otorga el rol de ciencia; entonces plantea enfocarlo desde el papel de las relaciones interdisciplinarias en el establecimiento de un posible objeto de estudio.

Lo cierto desde nuestro punto de vista es que la Criminalística, se enfoca en identificar cómo participan en la consumación de un hecho, presumiblemente delictivo, diferentes elementos físicos que dejan su marca cuando irrumpen en un escenario determinado. Para ello se auxilia del método científico como la vía para determinar de manera plausible la relación de las evidencias con el hecho bajo investigación. Como plantea Chávez, (2021, p.45): "Más allá de emplear el método científico, la criminalística es ciencia porque 
estudia resultados, resultados observables y tangibles de los eventos..." Chávez, sin embargo, circunscribe a la Criminalística a la búsqueda o verificación de pruebas a partir del análisis de evidencias en hechos delictivos, cuestión que se aprecia también en otros autores como Pesantez, Valarezo y Vilela (2021) quienes la consideran de utilidad para la determinación de la veracidad del delito. En nuestra opinión es mucho más actual y adaptada a las condiciones reales en las que debe y puede actuar la Criminalística lo planteado por Ccaza (2012) quien abre su radio de intervención al pronunciamiento pericial de información referente tanto a procesos de índole judicial, administrativo o particular, no necesariamente delictivos.

Un aspecto en el que coinciden diferentes autores García (2015), Pesantez, Valarezo y Vilela (2021) es el que se refiere al valor de la Criminalística para la aplicación de determinadas técnicas con el fin de obtener información e interpretarla para descubrir los elementos presentes en un hecho delictivo. Por tanto, desde la Criminalística se realizan las indagaciones sobre los hechos con el fin de identificar las evidencias que permitan la aceptación o refutación de las hipótesis formuladas acerca de ellos.

El concepto de evidencia ha sido también objeto de diferentes interpretaciones. Chávez (2021) insiste en que se debe establecer una cuidadosa diferenciación entre evidencias e indicios. Estos últimos apuntan a la posibilidad de que un elemento material participe en un determinado hecho, sin embargo, cuando este es identificado como interviniente en dicho suceso, se convierte en una evidencia. De cierta forma puede afirmarse que cuando el indicio adquiere carácter de prueba se funden indicio y evidencia y se convierten en guía para la interpretación de lo sucedido.

Las evidencias constituyen el elemento básico del trabajo pericial. A partir de ellas el experto pericial orienta su investigación para procesar las evidencias utilizando distintos métodos, con la finalidad de elaborar el informe pericial que será presentado ante el juez, quien lo utilizará como apoyo para dictar sentencia. De este modo para fundamentar cualquier tesis acerca de un caso, primeramente, se debe partir de las evidencias que prueban o rechazan la ocurrencia del suceso acerca del cual se plantea una teoría del caso. Por todo lo antes planteado el manejo de la evidencia física es sumamente importante para los estudios de criminalística, sin embargo, pese a los intentos realizados en el proceso de construcción de este elemento, existen todavía imprecisiones en el establecimiento de los protocolos necesarios para el correcto manejo de pruebas en el 
contexto específico de los hechos criminales; así como en el tratamiento posterior encaminado a que los objetos obtenidos como parte del estudio pericial, pasen a ser pruebas dentro de una audiencia de juicio oral, o sencillamente fortalecer la verosimilitud de una investigación y en consecuencia de la teoría del caso. Desde estos precedentes se realiza este trabajo cuyo objetivo general es contribuir a la divulgación de la importancia del manejo pertinente de las evidencias físicas en el trabajo pericial para una correcta formulación de la teoría del caso.

Los objetivos específicos son: 1) Caracterizar el proceso de manejo de las evidencias físicas que se producen en un hecho ilícito. 2) Describir cómo interviene la ciencia Criminalística en el esclarecimiento y comprensión de los hechos a partir del tratamiento de la evidencia física. 3) Explicar las relaciones existentes entre evidencia física y teoría del caso.

\section{MATERIALES Y MÉTODOS}

Autores como Guirao (2015), Terrero, Salazar y Toala (2019), plantean la importancia de los artículos de revisión por la síntesis y análisis crítico que realizan del estado del arte de un fenómeno en estudio. Según Reyes (2020, p. 103), un artículo de revisión es "un análisis retrospectivo de estudios compilados en la literatura sobre un tema que se considera interesante para un público general o especializado".

De acuerdo a estos criterios, en este trabajo se ha optado por la realización de un artículo de revisión acerca de las relaciones entre las evidencias físicas y la teoría del caso, partiendo de tres ideas básicas, cuya defensa contribuye a direccionar el esquema de la búsqueda de información, la recogida de datos y su discusión.

La primera idea a defender es: El manejo adecuado de las evidencias acerca del hecho delictivo respalda el establecimiento de la verdad en el proceso judicial.

La segunda idea a defender es que el esclarecimiento del papel de las evidencias obtenidas en un caso debe producirse desde la investigación científica.

Por último, la tercera idea que se defiende apunta a que la presentación de las evidencias identificadas en el informe pericial, apoya el planteamiento de la teoría del caso como herramienta de organización de las ideas desde un fundamento fáctico probatorio.

La búsqueda de documentos se realizó en Google Académico, empleando como términos de búsqueda: evidencia física y teoría del caso. La búsqueda utilizó como filtro a la disciplina, considerando materiales propios del Derecho y de la Criminalística. 
En la realización del proceso investigativo fueron empleados métodos del nivel teórico como el inductivo deductivo para identificar las premisas particulares que permitieron argumentar las ideas de partida, arribando a conclusiones y el analítico sintético en el develamiento de las particularidades de las relaciones entre los elementos en estudio: evidencia física y teoría del caso, alcanzadas en la revisión documental, que constituyó el método del nivel empírico utilizado para obtener la información.

\section{RESULTADOS Y DISCUSIÓN}

\section{EI manejo de evidencias en un hecho delictivo}

Uno de los fundamentos de un Estado de Derecho, lo constituye el cuidado y preservación de los derechos de los ciudadanos, incluso de aquellos que han cometido algún tipo de delitos. Es por eso que dentro de la normativa se fijan las garantías que permiten salvaguardar esos derechos. En el caso de Ecuador, por ejemplo, la Constitución como Ley Suprema del Estado establece que

"en todo proceso en el que se determinen derechos y obligaciones de cualquier orden, se asegurará el derecho al debido proceso que incluirá las siguientes garantías básicas... I) Las resoluciones de los poderes públicos deberán ser motivadas. No habrá motivación si en la resolución no se enuncian las normas o principios jurídicos en que se funda y no se explica la pertinencia de su aplicación a los antecedentes de hecho. Los actos administrativos, resoluciones o fallos que no se encuentren debidamente motivados se considerarán nulos. Las servidoras o servidores responsables serán sancionados". (Ecuador. Asamblea Nacional Constituyente, 2016).

A partir de este precedente cualquier fallo judicial debe estar adecuadamente fundamentado, lo que significa que su pertinencia debe estar motivada desde una adecuada descripción de los hechos, sustentada en un informe pericial a través del cual los peritos, como resultado de un proceso de investigación científica sobre los hechos, recopilan las evidencias y aportan las pruebas que le posibilitan al Juez determinar o rechazar la veracidad de un hecho delictivo.

Un hecho delictivo es un evento que al producirse genera consecuencias contrarias a las normas socialmente aprobadas, con lo cual se altera el orden vigente planteado por el Estado. Cuando se produce un hecho delictivo además de transgredirse determinadas 
normas sociales, se producen rupturas y alteraciones del escenario físico en el cual ocurren. Estos resultados son de interés para la Criminalística, la cual se enfoca en comprender los cambios que se producen en el espacio físico donde ocurre un delito en relación a su estado primario y cómo se conectan con el hecho ilícito.

Diferentes autores como Dasilva, Hernández, Hernao y Caicedo (2018) y Arnold (2021) se refieren a las evidencias físicas, Ramírez (2015) menciona que son “cualquier objeto relacionado con una conducta punible que puede servir para determinar las circunstancias reales de tiempo, modo y lugar en las que el hecho se realizó y que deberá someterse al régimen de cadena de custodia" (p.190), lo que puede interpretarse como aquella huella o marca palpable que fue dejada como rastro en el proceso de comisión del delito.

Se coincide con Restrepo (2005, p.38) quien define a la evidencia como el "conjunto de materiales, objetos y sustancias que guardan relación con el caso que se investiga, de diversa naturaleza y origen, dejados por la ejecución de la actividad delictiva, cuyo potencial radica en que sirvieron para cometer el hecho o consecuencia del mismo".

En el proceso de investigación criminalística, en una escena o lugar del hecho pueden ser encontradas numerosas de estas huellas o marcas, que inicialmente se consideran como indicios, o sea, como señales de que algo ha ocurrido allí. Por ejemplo, si hay un cuerpo ensangrentado y junto a él un cuchillo, este último es considerado un indicio de posible arma homicida. Cuando este indicio se procesa como resultado de haber ingresado a una cadena de custodia, pasa a ser considerado como una evidencia.

Ante la ocurrencia de un hecho delictivo, es imprescindible que se produzca un buen manejo de los indicios. Cualquier escena del crimen tiene evidencia física relacionada con lo sucedido en ella. Según plantean Mora y Sánchez (2014): “Toda escena de un crimen contiene evidencia física, la ausencia de ella es también evidencia” (p. 63). Mantener una cadena de custodia para los elementos físicos o materiales que constituyen indicios en un caso, reviste una gran importancia pues su estudio puede o no demostrar su carácter probatorio en un caso determinado.

El efecto probatorio de una evidencia se relaciona directamente con la calidad que haya tenido la cadena de custodia en la que fue incluida.

"En efecto, si para demostrar como cierto un hecho, se utiliza evidencia contaminada o adulterada cuya interpretación, en lugar de llevar a 
conclusiones veraces lleva al investigador y luego al juzgador a "dar por cierta" una mentira, para la justicia, la situación se torna peor que si no se hubiese presentado ningún tipo de evidencia, en ese juicio”. (Mora y Sánchez, 2014, p. 190)

En los últimos años han proliferado programas televisivos del estilo de Crime Scene Investigation (CSI), que han rodeado de una cierta aureola el trabajo del perito criminalista, sin embargo, la realidad no siempre es la que se observa en la serie, la cual recrea en especial las escenas de análisis especializado de las evidencias, en el lugar en que se produjo o en el laboratorio. Realmente el trabajo para alcanzar la autenticidad en el manejo de la escena de un hecho ilícito, no se inicia en un laboratorio sino con la observación inicial de la escena que realiza el experto criminalista, primero buscando la visión general del sitio y luego particularizando en los detalles. Es con posterioridad a esta observación general que se pasa a resguardar los indicios identificados, y se da cumplimiento a los trámites oficiales establecidos para la cadena de custodia con el fin de conservarlos, dentro de los límites de la legalidad. Cuando se han recolectado todos los indicios, se inicia el peritaje especializado que se concentra en manos de los especialistas, quienes son los encargados de analizar todos los indicios, interpretar las causas o el origen de lo ocurrido y precisar si es que son válidos como evidencia probatoria.

La criminalidad tiene un origen multicausal, en su aparición pueden estar presentes factores relacionados con la calidad de vida, el bienestar social, las cuestiones educativas, laborales, políticas y económicas, es por ello que, en la escena de un delito se pueden hallar diferentes indicios, los que directa o indirectamente pueden relacionarse con los determinantes que condujeron a su ocurrencia. Es precisamente esta multiplicidad causal que se halla en la génesis del acto ilícito, la que provoca que pueden ser igualmente variados los indicios presentes en el escenario de los hechos.

Las evidencias físicas pueden revelarse desde diferentes ángulos, por lo que hay varios tipos de evidencias: huellas, rastros, manchas, residuos, vestigios y similares, armas, dinero, bienes filmación, fotografía, video o cualquier otro medio avanzado. Estas evidencias se relacionan con el tipo de delito y gran parte del éxito en el proceso penal va a depender de que el manejo inicial de todos los materiales en la escena del delito sea 
realizado con total cuidado para que revele las causas y condiciones en que se produjo respetando el marco de derechos de las personas involucradas.

Sánchez (2013) reconoce que las evidencias físicas son muy importantes para probar un delito y que un buen proceso de identificación de las evidencias físicas, debe ser legal y auténtico:

La importancia de las evidencias físicas es que estas pueden probar la comisión de un delito, siempre cuando se realizan adecuadamente, porque para ello requiere ser acreditar tanto su legalidad como su autenticidad. Cuando se habla de su legalidad consiste en que su recolección u obtención se haya verificado observando el respeto a los derechos humanos en la forma establecida en la Constitución, en los tratados internacionales y en las leyes. Cuando se refiere a su autenticidad implica que su detención, fijación, recolección y embalaje se haya efectuado técnicamente y que se haya sometido a la cadena de custodia (p.4).

En el manejo de las evidencias físicas es fundamental que cualquier descuido sea evitado, so pena de que si no se cuidan puede llegarse a su alteración o destrucción. Como resultado del trabajo investigativo pericial las pruebas acerca de la comisión del hecho delictivo, adquieren relevancia penal. Es por eso que cualquiera de estos peritajes debe recibir un tratamiento muy cuidadoso, de modo que se garantice que, en el momento del juicio oral, la evidencia física que se presente no haya sido adulterada, ocasionando que pierda su utilidad y no se pueda confirmar o rechazar la responsabilidad penal.

En el Código Integral Penal (COIP) de Ecuador en su Art.456.- se establece la realización de la Cadena de Custodia, la cual inicia en el lugar donde se producen los hechos y es responsabilidad de todas las personas que tienen relación con su utilización para revelar el estado de los hechos.

\section{La investigación criminalística en el proceso de estudio de indicios}

Según García (2015) la investigación criminalística constituye una disciplina autónoma encargada de apoyar el ejercicio de la justicia auxiliándola a través del análisis de los indicios, con el uso de diferentes técnicas cuyo sustrato puede hallarse en otras disciplinas, como puede ser la biología, la medicina, la antropología, etc. 
De acuerdo con la opinión de Pesantez, Valarezo y Vilela (2021), esta investigación se considera como

[...] un conjunto de saberes interdisciplinarios y acciones sistemáticas integrados, para llegar al conocimiento de una verdad relacionada con el fenómeno delictivo; es el proceso tendiente a comprobar la existencia de un delito y la responsabilidad del imputado, para lo cual se requiere de peritos capaces. (p.445)

Existen autores como Lago (2017) que ligan la investigación criminalística a la conducta de los seres humanos, considerando que ha estado presente a lo largo de su evolución. En ese sentido considera que la Criminalística está orientada al establecimiento de la verdad sobre los hechos y a determinar la responsabilidad penal en su ejecución. Como en el caso de los autores mencionados al inicio del epígrafe, opina que la investigación criminal se auxilia de los aportes de otras ciencias y de métodos como la inducción, la deducción y la experimentación.

La investigación criminalística como cualquier proceso de investigación científica constituye un proceso riguroso y sistemático que se sustenta en la aplicación del método científico, por lo cual transita por las etapas en las que este se lleva a cabo. Es por ello que en una investigación criminalística se realiza un sucesivo acercamiento al estudio de su objeto: el delito, partiendo de la observación del lugar en el cual se produjo, el planteamiento de hipótesis, la recolección y procesamiento de la información que permite confirmarla o rechazarla y el establecimiento de las conclusiones que a través de un informe pericial serán presentadas para su uso en el correspondiente proceso judicial, con el fin de que el Estado administre justicia determinando y sancionando las conductas delictivas.

Diferentes autores se han referido al inicio del proceso de investigación criminal y existe coincidencia entre algunos de ellos como Constain (1962) y Campos (2002), en que este inicia con la inspección al escenario de los hechos, al cual le asignan la cualidad de ser el momento que puede garantizar la pureza de la información directamente relacionada con el suceso bajo estudio. Lo esencial del procedimiento utilizado en la recolección de indicios es contribuir en el proceso que permitirá transformarlo en evidencia.

A continuación, a partir de la consulta de la literatura revisada, se realiza una sistematización de las etapas en las que se organiza la investigación criminalística, a la 
cual en un lenguaje especializado se le reconoce como administración de la cadena de custodia. Es necesario señalar que, cualquiera de las pericias llevadas a cabo por la policía judicial o los peritos expertos señalados, se debe realizar con la presencia del fiscal, quien dirige el proceso desde la indagación previa hasta su última fase que es la de impugnación. En Ecuador esto último queda debidamente registrado en el Art. 458, numeral 5 del COIP. - La fijación y recolección de las evidencias, huellas, vestigios encontrados en el lugar ingresarán en cadena de custodia para la investigación a cargo de la o el fiscal, quien dispondrá las diligencias pertinentes.

1) La fijación del lugar de los hechos: En esta etapa se produce la apertura del proceso de investigación, con la llegada al lugar donde se produjeron los hechos. En ese momento es fundamental la preservación del lugar, para evitar que se produzca una contaminación de los indicios que arrojen información importante sobre el caso. En la misma existen algunas acciones que son fundamentales, como son:

- Controlar la hora del suceso, las personas que estén presentes y notificar a quienes deban conocer de los hechos. En este primer momento pueden ser realizadas las primeras entrevistas tendientes al esclarecimiento primario de lo ocurrido, acción que en ocasiones está en manos de la policía judicial, en otros casos se encomienda a personal con experticia en la realización de interrogatorios en la escena del delito, la cual en ese primer momento obedece generalmente a la identificación de las condiciones de tiempo, modo y lugar en que sucedieron los hechos.

- Controlar que no se produzcan cambios en el lugar de los hechos que alteren cualquier indicio posible, como huellas dactilares o de pasos.

- No permitir que permanezcan en el lugar las personas que no tengan relación con el hecho.

- Organizar a los técnicos que estén en el lugar con fines de investigar.

Esta primera etapa concluye con la preparación de los informes respectivos y la entrega de dichos elementos para su resguardo.

2) Realización de las diligencias encaminadas a la reserva, empacado y se rubrican los elementos que se identificaron en el lugar de los hechos para su conservación y cuidado.

3) Decisión del destino de los indicios recolectados: Esto implica remitirlos a un lugar de almacenamiento o a un laboratorio para ser estudiados. 
4) Análisis de las pruebas recolectadas a nivel de laboratorio especializado: En esta etapa el trabajo fundamental se concentra en las manos de los peritos forenses, encargados de determinar si los indicios se relacionan con los hechos investigados. Es el momento en el cual los indicios se convierten en evidencias, las cuales puedan argumentar con base científica la responsabilidad de determinadas personas en la ocurrencia de un delito. A nivel de laboratorio los estudios realizados guardan relación con el tipo de delito bajo análisis, así pueden encontrarse, pruebas caligráficas, dactiloscópicas, químicas, etc. a partir de las cuales se agruparán las evidencias.

5) Elaboración del informe con los resultados periciales y entrega al Fiscal que se ocupa del caso.

Al hacer referencia a estas etapas, puede tal vez entenderse que el paso por ellas se produce cíclicamente, sin embargo, pese a ser un proceso de extrema organización, pueden producirse caminos intermedios que viabilicen algunas de sus acciones, por ejemplo, la no existencia de un laboratorio especializado cerca del lugar donde ocurren los hechos, puede implicar que estos deban permanecer hasta tanto sea posible su estudio en un almacén transitorio, sin embargo, esto no puede alterar la calidad de la investigación.

Es aquí donde se refuerza la consideración de la cientificidad de la criminalística, lo que implica que la investigación de los hechos en cualquier situación delictiva debe ir acompañada de la rigurosidad suficiente en el manejo técnico científico de las pruebas necesarias para identificar a sus autores y los procedimientos que indiquen su nivel de responsabilidad. La ciencia Criminalística, según Ccaza (2012, p. 82) "[...] desde su génesis, se ha nutrido de múltiples aportes derivados de las ciencias, las artes y la técnica, que han coadyuvado a enfrentar el reto de responder a una serie de interrogantes clave en la reconstrucción de los hechos que ésta investiga”. Así la Criminalística se acompaña de un conjunto de disciplinas que le ayudan a delinear su estatus científico como son la balística forense, la fotografía forense, técnicas forenses de laboratorio (química, física, biología), etc.

En la actualidad se considera que los resultados de la investigación criminalística son fundamentales, aunque no únicos, en el establecimiento de la verdad. Como cualquier otro proceso de investigación, es susceptible para la verificación de sus conclusiones, por lo que es práctica común que, en un juicio oral, no se haga lectura del informe pericial, 
sino que se reconstruya la prueba pericial dentro de la audiencia del juicio oral, cuando se somete al perito forense en su calidad de experto al interrogatorio por las partes implicadas, según expresa León (2014).

\section{Incorporación de las evidencias a la teoría del caso}

En los países que cuentan con un sistema penal acusatorio, existen diferentes herramientas metodológicas que están al servicio del proceso penal y contribuyen a su desenvolvimiento, una de ellas es la denominada teoría del caso, la que tiene que ver con la organización del pensamiento lógico jurídico tanto de la parte acusatoria como de la defensa. Cazares y Guillén (s/f), le adjudican el rol de guion de lo que será demostrado en el juicio mediante el uso de las pruebas.

Sería imposible pensar en establecer una teoría del caso durante la realización de un juicio oral si no se incorporan a este las pruebas que respalden cualquier acusación. Las evidencias recopiladas durante el proceso previo de preparación de la audiencia oral, en la etapa de instrucción policial y producto del trabajo de los expertos peritos, adquieren al ser incorporadas al juicio, el carácter de pruebas.

Autores como Cafferata (1998, p. 224), el proceso penal se origina en la necesidad de descubrir la verdad sobre la hipótesis delictiva que se ha construido, lo que solo puede ser logrado con las pruebas que apoyen el trabajo con esta hipótesis. Sánchez (2009), estima que las pruebas son el vehículo que permite llegar a la verdad, aspecto en el cual también coincide González (2015).

En el Código Integral Penal del Ecuador (COIP), el uso de las pruebas se refleja en el Art. 453.- Finalidad. - La prueba tiene por finalidad llevar a la o al juzgador al convencimiento de los hechos y circunstancias materia de la infracción y la responsabilidad de la persona procesada.

El uso de las pruebas tiene un carácter de obligatoriedad, incluso en los casos en que la persona se considere culpable, el Fiscal no queda liberado de efectuar la práctica de pruebas, como se establece en el Art. 509 del COIP.

Los principios del anuncio y la práctica de las pruebas se especifican en el Art. 454.- 
Tabla 1. Principios del anuncio y práctica de la prueba

\begin{tabular}{|c|c|}
\hline Principios & Contenido del principio \\
\hline 1. Oportunidad & $\begin{array}{l}\text { Es anunciada en la etapa de evaluación y preparatoria de juicio y se } \\
\text { practica únicamente en la audiencia de juicio. }\end{array}$ \\
\hline 2. Inmediación & $\begin{array}{l}\text { Las o los juzgadores y las partes procesales deberán estar presentes } \\
\text { en la práctica de la prueba. }\end{array}$ \\
\hline 3. Contradicción & $\begin{array}{l}\text { Las partes tienen derecho a conocer oportunamente y controvertir } \\
\text { las pruebas, tanto las que son producidas en la audiencia de juicio } \\
\text { como las testimoniales que se practiquen en forma anticipada. }\end{array}$ \\
\hline $\begin{array}{l}\text { 4. Libertad } \\
\text { probatoria }\end{array}$ & $\begin{array}{l}\text { Todos los hechos y circunstancias pertinentes al caso, se podrán } \\
\text { probar por cualquier medio que no sea contrario a la Constitución, } \\
\text { los instrumentos internacionales de derechos humanos, los } \\
\text { instrumentos internacionales ratificados por el Estado y demás } \\
\text { normas jurídicas. }\end{array}$ \\
\hline 5. Pertinencia & $\begin{array}{l}\text { Las pruebas deberán referirse, directa o indirectamente a los hechos } \\
\text { o circunstancias relativos a la comisión de la infracción y sus } \\
\text { consecuencias, así como a la responsabilidad penal de } \\
\text { la persona procesada. }\end{array}$ \\
\hline 6. Exclusión & $\begin{array}{l}\text { Toda prueba o elemento de convicción obtenidos con violación a } \\
\text { los derechos establecidos en la Constitución, en los instrumentos } \\
\text { internacionales de derechos humanos o en la Ley, carecerán de } \\
\text { eficacia probatoria, por lo que deberán excluirse de la actuación } \\
\text { procesal. }\end{array}$ \\
\hline $\begin{array}{l}\text { 7.Igualdad de } \\
\text { oportunidades } \\
\text { para la prueba }\end{array}$ & $\begin{array}{l}\text { Se deberá garantizar la efectiva igualdad material y formal de los } \\
\text { intervinientes en el desarrollo de la actuación procesal. }\end{array}$ \\
\hline
\end{tabular}

Tomado del Código Integral Penal (Versión del 17 de febrero del 2021)

La valoración de las pruebas incluye tanto aspectos de carácter formal, como algunos que se relacionan con el nivel tecno científico y la autenticidad que poseen. El propio COIP establece los principales medios de prueba en su Art. 498.- 1. El documento, 2. El testimonio y 3. La pericia. 
Para poder formular la teoría del caso se hace necesario un conocimiento lo más completo posible sobre los hechos, con el fin de proyectar la dirección que seguirá el proceso judicial y cómo será formulada y enfrentada la acusación. La teoría del caso es el medio idóneo para el trabajo encaminado a ubicar las evidencias en el lugar que le corresponde dentro del esquema de los hechos, determinando el lugar preciso de cada prueba y cómo se argumenta cada aspecto en la teoría manifestada.

\section{El establecimiento de la teoría del caso se sostiene en tres pilares fundamentales:}

a) Fáctico: Se refiere a la reconstrucción de pruebas durante el debate oral, lo que permite revelar los hechos según las condiciones de tiempo, lugar y el modo en que se produjeron, b) Jurídico: Tiene su base en la consideración de las normas penales dentro de las cuales se enmarca el caso,

b) Probatorio: Se realiza desde la parte acusatoria con la presentación de las pruebas convenientes que demuestren la culpabilidad del acusado y de la defensa con las correspondientes evidencias de descargo que lo liberan de culpas. En ambos casos se debe demostrar ante el Juez el punto de vista de cada parte.

De este modo una buena teoría del caso debe abarcar la mayor cantidad posible de los hechos presentes en el caso y ofrecer una explicación para ellos, de ahí la importancia que tiene el manejo de evidencias probatorias, las cuales se convierten en el punto de inicio y de llegada en el proceso, le dan una dirección a las diferentes diligencias y actividades procesales y sostienen la verificación de las hipótesis que sobre el caso esgrimen las partes.

En el caso específico de Ecuador, Espinoza (2010) manifiesta que aún existen vacíos en el establecimiento de relaciones entre los hechos y el Derecho, para alcanzar una real motivación de las decisiones judiciales. Uno de esos vacíos ese refiere a la ausencia de motivación bien establecida para los hechos. Si para considerar un hecho motivado, la fundamentación en derecho debe fijarlos, la descripción fáctica es un requisito indispensable para motivar una sentencia y muchas veces se carece en los procesos judiciales de una valoración crítica de las pruebas incorporadas, las cuales son únicamente mencionadas y se agota con esto su alcance para establecer una sentencia.

\section{CONSIDERACIONES FINALES}

La criminalística se interesa en los cambios que ocurren en el lugar donde se ha producido un ilícito, con el fin de estudiarlos, descubrir cómo se relacionan con ese hecho e informar 
a los órganos competentes para que sean tomadas las medidas pertinentes. Este es el proceso encaminado a generar las evidencias probatorias, sin cuya participación le sería muy difícil a los magistrados demostrar el nivel de responsabilidad de los participantes. Para que este proceso fluya auténticamente se precisa de un riguroso trabajo técnico científico con las evidencias obtenidas en cada caso, dado que el efecto probatorio de cualquier evidencia depende directamente de la calidad que haya tenido la cadena de custodia en la que fue incluida.

Las evidencias se relacionan con el tipo de delito en cuyo escenario se identifican primeramente como indicios, gran parte del éxito en el proceso penal depende de que el manejo de todos los materiales en la escena del delito sea realizado con total cuidado desde el principio, buscando que revele las causas y condiciones en que se produjo, siempre dentro del marco de derechos de las personas involucradas.

Para el estudio de su objeto, la Criminalística se acompaña de un conjunto de disciplinas que le ayudan a delinear su estatus científico, apegándose estrictamente en su práctica a una metodología cuyo sustento se alcanza a través de la aplicación del método científico en sus diferentes etapas; reconociendo que los errores que se cometan en el análisis y procesamiento de los datos periciales pueden llevar a fallos en las decisiones judiciales que perjudiquen el derecho de los acusados al debido proceso.

El manejo de las evidencias probatorias constituye uno de los pilares en el establecimiento de la teoría del caso, exigiendo un conocimiento lo más completo posible sobre los hechos, con el fin de proyectar la dirección en que se han de realizar las diferentes diligencias y actividades procesales, para la verificación de las hipótesis que sobre el caso esgrimen las partes.

En el caso de Ecuador, aunque desde el COIP están establecidas las maneras de regular el uso de las pruebas, aun existen vacíos en su incorporación y argumentación en el proceso judicial, aspecto que deberá ser resuelto para permitir de manera adecuada que el Juez demuestre los hechos, fundamentándolos en derecho, para lo cual debe describirlos, calificarlos, y subsumirlos desde la correspondiente norma jurídica.

\section{LISTA DE REFERENCIAS}

Arnold, D. (2021). Las evidencias y pruebas como fundamento de validez científica en las auditorías forenses. Revista Colón Ciencias, Tecnología y Negocios, vol. 8, núm. 2. 
Campos, F. (2002). Cadena de custodia de la prueba. Primera edición, Costa Rica: Editorial Jurídica Continental.

Cazares. O. F. y Guillén, D (s/f), Teoría del caso en el sistema penal acusatorio. http://www.juridicaformativa.uson.mx/memorias/v_coloquio/doc/derechoconstit ucional/CAZAREZ_OLGA_Y_GERMAN_GUILLEN.pdf

Ccaza, J. E. (2012). Criminalística y Derecho Probatorio en Materia Penal. Grupo EDITORIAL CROMEO S.A.C.

Cafferata, J. (1998). La puerta en el proceso penal. Buenos Aires: Editorial Depalma,

Chávez, C. (2021). Criminalística práctica: La evidencia. teoría y procesamiento físico. Gac. int. Cienc. Forense, № 39. abril-junio.

Constain, M. y Constain, A. (1963). Investigación Criminal. Bogotá, Colombia: Editorial Temis Librería.

Dasilva, S., Hernández, L., Hernao, N., y Caicedo, A. (2018). La calidad de la evidencia física: análisis frente a valoración del riesgo. Revista Sinergia, 1(1), 171-198.

Ecuador. Asamblea Nacional Constituyente. (2016). Constitución de la República del

Ecuador. http://www.asambleanacional.gob.ec/sites/default/files/documents/old/constituci on_de_bolsillo.pdf

Ecuador. Asamblea Nacional. (2021). Código Orgánico Integral Penal. Registro Oficial No. $\quad 180 . \quad$ https://www.defensa.gob.ec/wpcontent/uploads/downloads/2021/03/COIP_act_feb-2021.pdf

Ecuador. Consejo Nacional de la Judicatura. (2017). Reglamento del Sistema Pericial Integral de la Función Judicial. (Modificación). Registro Oficial Edición Especial 125. Quito: CNJ

Elbert, C. (1988). Criminología Latinoamericana. Teoría y propuestas sobre el control social del tercer milenio. Editorial Universidad, Buenos Aires, 1996.

Espinoza, C. (2010). Teoría de la motivación de las resoluciones judiciales y jurisprudencia de casación y electoral. Tribunal Contencioso Electoral. V\&M GRAFICAS.

García, F. (2015). Principios Constitucionales Fundamentales del Derecho Procesal Ecuatoriano. https://www.derechoecuador.com/principios$\underline{\text { constitucionales-fundamentales-del-derecho-procesal-ecuatoriano }}$ 
González, D. (2015). Presunción de inocencia, verdad y objetividad. Materiales del Máster en Argumentación Jurídica, Universidad de Alicante, http://rua.ua.es/dspace/handle/10045/46907

Guirao G. y Silamani J. A. (2015). Utilidad y tipos de revisión de literatura. ENE, Revista de Enfermería. v. 9, n. 2, ago. http://ene.enfermeria.org/ojs

Lago, V. (2017). La práctica de la investigación criminal: Inspección Técnico Ocular (ITO). Editorial Reus S.A, 184 págs.

León, D. (2014). Del indicio a la evidencia en criminalística y criminología. para la acusación de un homicida. Tesis de Diplomado en investigación criminal para el sistema penal acusatorio. Universidad de la Gran Colombia.

López P., (2008). Investigación criminal y criminalística en el sistema penal acusatorio. Bogotá D.C. Temis.

Mora, R. y Sánchez, M. (2014). La Evidencia Física y La Cadena de Custodia en el Procedimiento Acusatorio. Bogotá. Editores Gráficos Colombia S.A.S

Pesantes, L. E., Valarezo, L. H., y Vilela, W. E. (2019). Importancia de la investigación judicial y criminalística en la determinación de la veracidad del delito. Universidad y Sociedad, 11(4), 443-449. Recuperado de http://rus.ucf.edu.cu/index.php/rus

Ramírez, M. y Bohórquez, J. R. (2013). Metodología y desarrollo de la auditoría forense en la detección del fraude contable en Colombia. Cuadernos de Administración, Universidad del Valle, Vol. $29 \mathrm{~N}^{\circ}$ 50, 186-195.

Reyes, H. (2020). Artículos de Revisión. Revista médica de Chile, 148(1), 103108. https://dx.doi.org/10.4067/S0034-98872020000100103

Restrepo, M. (2005). El Nuevo sistema acusatorio. Bogotá, Colombia, Edit. Intermedio.

Sánchez, F. P. (2013). Reflexiones sobre manejo adecuado de cadena de custodia en nuevo proceso penal. Revista Jurídica del Centro, Vol 4. http://www.cartapacio.edu.ar/ojs/index.php/RJC/article/view/1430/1679

Terreros, M. A.; Salazar, J.; Toala, A. (2019). Artículo de revisión. Fases de elaboración y protocolo. Revista Científica Universidad Odontológica Dominicana. Vol. 7. $\mathrm{N}^{\circ} 1$. 